\title{
BOUNDS FOR NEARLY BEST APPROXIMATIONS
}

\author{
RUDOLF WEGMANN
}

ABSTRACT. Let $X$ be a uniformly convex space and $\psi$ be the inverse function of the modulus of convexity $\delta(\cdot)$. Assume here that $\psi$ is a concave function. Let $V$ be a linear subspace of $X$ and let $f$ in $X$ be such that $\|f\|$ $=1=\min \{\|f-v\|: v \in V\}$. Then for $0<\delta \leq 1$ and for $v$ in $V$ with $\|f-v\| \leq$ $1+\delta$, it follows that $\|v\| \leq K \cdot \psi(\delta)$.

Let $T$ be a compact Hausdorff-space and $V$ a finite-dimensional subspace of $C(T, X)$. When $V$ has the interpolation property $\left(P_{m}\right)$ with $\operatorname{dim} V=$ $m \cdot \operatorname{dim} X$, then the same type of estimate as above holds.

Let $X$ be a uniformly convex normed linear space [1], i.e., for each $\epsilon$ with $0<\epsilon \leq 2$ there exists a $\delta(\epsilon)>0$ such that $x, y \in X,\|x\| \leq 1,\|y\| \leq 1$, and $\|x-y\|>\epsilon$ imply $\|(x+y) / 2\| \leq 1-\delta(\epsilon)$. The function $\delta(\cdot)$ is called the modulus of convexity of $X$. Without loss of generality we shall always assume that $\delta($.$) is monotone nondecreasing. Then an inverse function \psi$ can be defined by

$$
\psi\left(\delta_{0}\right):=\sup \left\{\epsilon: 0<\epsilon \leq 2, \delta(\epsilon)<\delta_{0}\right\}
$$

for $\delta_{0}>0$. Obviously, $\psi$ is monotone nondecreasing. From $\delta(\epsilon) \leq \epsilon / 2$ it follows that $\psi\left(\delta_{0}\right)>0$ for $\delta_{0}>0$.

One can replace $\delta(\cdot)$ by a monotone increasing convex function $\delta_{1}(\cdot)$, such that $0<\delta_{1}(\epsilon)<\delta(\epsilon)$ for $0<\epsilon \leq 2$ and $1 \leq \lim \inf _{\epsilon \rightarrow 0} \delta(\epsilon) / \delta_{1}(\epsilon)<\infty$. Then $\psi$ is concave and continuous.

Let $V$ be a subspace of $X$, and let $f$ be in $X$ such that $\|f\|=1$ and 0 is the best approximation for $f$ by elements of $V$. A question of some practical interest is that of how fast the "nearly best approximations" $v$ in $V$, with $\|f-v\| \leq 1+\delta$, approach 0 when $\delta \rightarrow 0$.

This note considers also the analogous question for subspaces $V$ of $C(T, X), T$ compact, and gives estimates for $\|\nu\|$ in terms of the function $\psi$.

Theorem 1. The diameter $D(C)$ of every convex subset $C$ of the spherical shell $R(\delta):=\{x \in X: 1-\delta \leq\|x\| \leq 1\}$ is $\leq \psi(\delta)$.

A result of this type was given by Fan and Glicksberg [2], but they did not relate the bound on $D(C)$ to the modulus of convexity.

Proof. From (1) it follows that $\delta(\epsilon) \geq \delta_{0}$ for $\epsilon>\psi\left(\delta_{0}\right)$. So, $\|x\| \leq 1$, $\|y\| \leq 1$, and $\|(x+y) / 2\|>1-\delta$ imply $\|x-y\| \leq \psi(\delta)$.

Received by the editors July 2, 1974.

AMS (MOS) subject classifications (1970). Primary 41A50; Secondary 41A65, $41 \mathrm{~A} 10,30 \mathrm{~A} 82$. 
Let $C$ be a convex subset of $R(\delta)$. Then for $x, y \in C, x \neq y$, the segment $[x, y]$ is in $R(\delta)$. Define $y_{\theta}:=x+\theta(y-x)$. Then $\|x\| \leq 1,\left\|y_{\theta}\right\| \leq 1$, and $\left\|\left(x+y_{\theta}\right) / 2\right\| \geq 1-\delta$ for $0 \leq \theta \leq 1$. Since $X$ is uniformly convex this last inequality is strict for all $\theta$ with at most one exception $\theta_{0}$. Thus we obtain $\left\|x-y_{\theta}\right\| \leq \psi(\delta)$ for all $\theta \neq \theta_{0}$ and by continuity also for $\theta_{0}$. Since $x$, $y$ in $C$ are arbitrarily chosen, $D(C) \leq \psi(\delta)$ is proved.

Theorem 2. Let $V$ be a linear subspace of $X$, let $f$ be in $X$ such that $\|f\|=1=\min \{\|f-v\|: v \in V\}$. Then for $0<\delta \leq 1$ and all $v \in V$ with $\|f-v\|$ $\leq 1+\delta$ it follows that $\|v\| \leq 2 \psi(\delta)$.

Proof. The set $C:=\{v \in V:\|f-v\| \leq 1+\delta\}$ is a convex subset of the shell $\{x \in X: 1 \leq\|x-f\| \leq 1+\delta\}$. Using Theorem 1 to estimate the diameter of $C$, we obtain

$$
\begin{aligned}
\|v\|=\|v-0\| & \leq D(C) \leq(1+\delta) \psi(1-1 /(1+\delta)) \\
& =(1+\delta) \psi(\delta /(1+\delta)) \leq 2 \psi(\delta) .
\end{aligned}
$$

Let $P_{V}$ be the metric projection on $V$, i.e. the mapping which assigns to each $f$ in $X$ its best approximation $P_{V}(f)$ by elements of $V$. It is well known that $P_{V}$ is uniformly continuous on bounded sets [5, p. 17]. From Theorem 2 we can obtain bounds for the modulus of continuity of $P_{V}$.

Corollary 1. Let $V$ be a linear subspace of $X$. Let $f, g$ in $X$ be such that $2\|f-g\| \leq E(f):=\min \{\|f-v\|: v \in V\}$. Then

$$
\left\|P_{V}(f)-P_{V}(g)\right\| \leq 2 E(f) \psi(2\|f-g\| / E(f)) .
$$

Proof. Without loss of generality we assume $P_{V}(f)=0$, so that $E(f)=$ $\|f\|$. Using $\left\|P_{V}(g)-g\right\| \leq\|f-g\|+E(f)$ we can estimate

$$
E(f) \leq\left\|P_{V}(g)-f\right\| \leq\left\|P_{V}(g)-g\right\|+\|f-g\| \leq E(f)+2\|f-g\| .
$$

It follows that

$$
1 \leq\left\|P_{V}(g)-f\right\| / E(f) \leq 1+2\|f-g\| / E(f),
$$

and by Theorem 2 ,

$$
\left\|P_{V}(g)-P_{V}(f)\right\| \leq 2 E(f) \cdot \psi(2\|f-g\| / E(f)) .
$$

Let $T$ be a compact Hausdorff space and $C(T, X)$ be the space of continuous functions $f: T \rightarrow X$ provided with the maximum norm $\|f\|:=$ $\max \left\{\|f(t)\|_{X}: t \in T\right\}$. A subspace $V$ of $C(T, X)$ is said to have the interpolation property $\left(P_{m}\right)$ if for every $m$ distinct points $t_{1}, \ldots, t_{m}$ in $T$ and elements $y_{1}, \ldots, y_{m}$ in $X$ there exists $v$ in $V$ such that $v\left(t_{i}\right)=y_{i}$ for $i=1$, $\ldots, m[6$, p. 201]. When the real dimensions are in the relation $\operatorname{dim} V=$ $m \cdot \operatorname{dim} X$, then there exists exactly one such $v$, and each function $v$ in $V$ which vanishes at $m$ distinct points on $T$ vanishes identically.

The following theorem is analogous to Theorem 2.

Theorem 3. Let $V$ be a linear subspace of $C(T, X)$ which has property 
$\left(P_{m}\right)$ with $\operatorname{dim} V=m \cdot \operatorname{dim} X$. Let $f$ in $C(T, X)$ be such that $\|f\|=1=$ $\min \{\|f-v\|: v \in V\}$. Then there exist numbers $K_{1}>0, K_{2} \geq 1$ depending on $f$ and $V$ such that for all $v$ in $V$ with $\|f-v\| \leq 1+\delta$ it follows that

$$
\|v\| \leq K_{1} \psi\left(K_{2} \delta\right)
$$

If $\psi$ is a concave function, then

$$
\|v\| \leq K_{3} \psi(\delta) \text {. }
$$

Proof. Let $n$ be the dimension of $V$ over the real field. According to $[6$, p. 202] the element 0 is a best approximation for $f$ by elements of $V$ if and only if there exist extremal points $x_{1}^{*}, \ldots, x_{h}^{*}$ of the unit ball $\left\{x^{*} \in X^{*}\right.$ : $\left.\left\|x^{*}\right\| \leq 1\right\}$ of the dual space $X^{*}$, points $t_{1}, \ldots, t_{h}$ in $T$ and positive numbers $\bar{\lambda}_{j}$ with $\Sigma_{j=1}^{h} \lambda_{j}=1$ such that

$$
\begin{aligned}
& \left.\sum_{j=1}^{h} \lambda_{j} x_{j}^{*}\left(v_{j}\right)\right)=0 \quad \text { for each } v \text { in } V, \\
& x_{j}^{*}\left(f\left(t_{j}\right)\right)=\|f\|=1 \quad \text { for } j=1, \ldots, h .
\end{aligned}
$$

The number $h$ is in the range $m+1 \leq h \leq n+1$.

Since $V$ has property $\left(P_{m}\right)$ with $\operatorname{dim} V=m \cdot \operatorname{dim} X$ from $v \in V$ and $v\left(t_{j}\right)=0$ for $j=1, \ldots, h$ it follows that $v \equiv 0$. Hence $\max \left\{\left\|v\left(t_{j}\right)\right\|: j=1\right.$, $\ldots, h\}$ is a norm on $V$. Since $V$ has finite dimension this norm is equivalent to the original one, i.e., there is a constant $K_{4}$ so that

$$
\|v\| \leq K_{4} \max \left\{\left\|v\left(t_{j}\right)\right\|: j=1, \ldots, h\right\} \text { for } v \text { in } V \text {. }
$$

From $\left\|f\left(t_{j}\right)-v\left(t_{j}\right)\right\| \leq 1+\delta$ it follows that $\left|x_{j}^{*}\left(f\left(t_{j}\right)-v\left(t_{j}\right)\right)\right| \leq 1+\delta$. For each fixed index $k$ in $1 \leq k \leq h$ we obtain from (4) and (5)

$$
\begin{aligned}
\sum_{j \neq k} \lambda_{j}+\lambda_{k} x_{k}^{*}\left(v\left(t_{k}\right)\right) & =\sum_{j \neq k} \lambda_{j} x_{j}^{*}\left(f\left(t_{j}\right)-v\left(t_{j}\right)\right) \\
& \leq \sum_{j \neq k} \lambda_{j}\left|x_{j}^{*}\left(f\left(t_{j}\right)-v\left(t_{j}\right)\right)\right| \leq \sum_{j \neq k} \lambda_{j}(1+\delta),
\end{aligned}
$$

and consequently

$$
\lambda_{k} x_{k}^{*}\left(\nu\left(t_{k}\right)\right) \leq\left(\sum_{j \neq k} \lambda_{j}\right) \delta .
$$

The number $K_{5}:=\max \left\{\Sigma_{j \neq k}\left(\lambda_{j} / \lambda_{k}\right): k=1, \ldots, b\right\}$ depends on $f$ and $V$, but not on $v$. So we obtain from (7)

$$
x_{k}^{*}\left(\nu\left(t_{k}\right)\right) \leq K_{5} \cdot \delta \text { for } k=1, \ldots, h .
$$

For both points, $x_{k}=0$ and $x_{k}=v\left(t_{k}\right)$, we have $\left\|f\left(t_{k}\right)-x_{k}\right\| \leq 1+\delta$ and $x_{k}^{*}\left(x_{k}\right) \leq K_{5} \cdot \delta$, hence by (5) $x_{k}^{*}\left(f\left(t_{k}\right)-x_{k}\right) \geq 1-K_{5} \delta$. Consequently $\left(f\left(t_{k}\right)-x_{k}\right) /(1+\delta)$ is in the convex subset $C:=\left\{x \in X:\|x\| \leq 1, x_{k}^{*}(x) \geq\right.$ 
$\left.\left(1-K_{5} \delta\right) /(1+\delta)\right\}$ of the spherical shell $\left\{x \in X: 1-\left(K_{5}+1\right) \cdot \delta /(1+\delta) \leq\right.$ $\|x\| \leq 1\}$. Using the estimate of Theorem 1 for the diameter $D(C)$ we obtain

$$
\left\|v\left(t_{k}\right)\right\| \leq(1+\delta) D(C) \leq(1+\delta) \psi\left(\frac{\left(K_{5}+1\right) \delta}{1+\delta}\right) \leq 2 \psi\left(\left(K_{5}+1\right) \delta\right)
$$

for $k=1, \ldots, h$. Together with (6) this yields the estimate (2). If $\psi$ is a concave function then we can use $\psi(\lambda \delta) \leq \lambda \psi(\delta)$ for $\lambda \geq 1$ to obtain (3).

For $X$ a real Hilbert space one can choose $\psi(\delta)=\delta$ if $\operatorname{dim} X=1$ and $\psi(\delta)=$ $2 \delta^{1 / 2}$ if $\operatorname{dim} X \geq 2$. We note that $\mathbf{C}$ is norm-isomorphic to the Euclidean $\mathbf{R}^{2}$. The space $V$ of the polynomials of degree $\leq n$ has the interpolation property $\left(P_{n+1}\right)$ in the real as well as in the complex case. So we obtain from Theorem 3 the following

Corollary 2. Let $T$ be a compact subset of $\mathbf{R}$ (or $\mathbf{C}$ ) with at least $n+2$ points and let $V$ be the space of polynomials of degree $\leq n$ restricted to $T$. Let $f$ be in $C(T, \mathbf{R})$ (or $C(T, \mathbf{C})$ ) such that $\|f\|=1=\min \{\|f-v\|, v \in V\}$. Then there exists a number $K$ dependent on $T, n$ and $f$, such that for all $v$ in $V$ with $\|f-v\| \leq 1+\delta$ it follows that $\|v\| \leq K \cdot \delta$ (or $\left.\|v\| \leq K \delta^{1 / 2}\right)$.

In the real case this is a result of Freud [3]. The complex case improves a result of Poreda, who proved in [4] only $\|v\|=O\left(\delta^{\beta}\right)$ for $0<\beta<1 / 2$.

Now we show that the estimate (3) is sharp in the sense that the function $\psi$ may not be replaced by another one $\psi_{1}$ such that $\psi_{1}(\delta) / \psi(\delta) \rightarrow 0$ as $\delta \rightarrow 0$. We make the hypothesis that $\psi$ is concave and sharp in the following sense: There exists a constant $K>0$ such that for all $x$ in $X$ and $x^{*} \in X^{*}$ with $\left\|x^{*}\right\|=1=\|x\|=x^{*} x$, from $\|y\|=1+\delta$ and $x^{*}(x-y)=0$ it follows that $\|y-x\| \geq K \psi(\delta)$.

We note that Hilbert-spaces have this property, when $\psi$ is specified as before Corollary 2. So the estimates of the corollary are sharp.

To prove the sharpness of (3) we proceed in the following way. Let $V$ be a subspace of $C(T, X)$ as in Theorem 3. We construct a suitable $f$ in $C(T, X)$ which fulfills the hypotheses of Theorem 3 such that for all $\delta>0$ there exists $v$ in $V$ such that $\|f-v\| \leq 1+\delta$ and $\|v\| \geq K \psi(\delta)$.

Let $t_{1}, \ldots, t_{m+1}$ be different points of $T$. The mapping $v \rightarrow\left(v\left(t_{1}\right)\right.$, $\left.\ldots, v\left(t_{m+1}\right)\right)$ carries $V$ onto an $n$-dimensional subspace of the $(m+1)$-fold product $W:=X \times \cdots \times X$, which has dimension $(m+1) \cdot \operatorname{dim} X>n$. So there exists a nontrivial linear functional $w^{*}$ on $W$ which vanishes on the image of $V$. Hence there exist $x_{j}^{*}$ in $X^{*}$ and real $\lambda_{j}$ such that

$$
\sum_{j} \lambda_{j} x_{j}^{*}\left(v\left(t_{j}\right)\right)=0
$$

for all $v$ in $V$. By suitable normalization we can reach $\left\|x_{j}^{*}\right\|=1, \lambda_{j} \geq 0$, $\Sigma \lambda_{j}=1$.

Since $X$ has finite dimension there exist $x_{j}$ in $X$ so that $\left\|x_{j}\right\|=1=$ 
$x_{j}^{*} x_{j}$. We put $f\left(t_{j}\right)=x_{j}$ and extend $f$ to an element of $C(T, X)$ such that for an $\eta>0$

$$
\|f(t)-v(t)\| \leq \max \left\{\left\|f\left(t_{j}\right)-v\left(t_{j}\right)\right\|: j=1, \ldots, m+1\right\}
$$

holds for all $t$ in $T$ and $v$ in $V$ with $\|v\| \leq \eta$. We omit the lengthy but elementary details of this construction. For this $f$ we have $\|f\|=1=\min \{\|f-v\|$ : $v \in V\}$.

If $X=\mathbf{R}$, it follows from $\|f-v\|=1+\delta$ that $\left|f\left(t_{j}\right)-v\left(t_{j}\right)\right|=1+\delta$ for at least one $j$, and so $\|v\| \geq\left|v\left(t_{j}\right)\right|=\delta$.

In case $\operatorname{dim} X \geq 2$ we construct a $v \neq 0$ in $V$ with

$$
x_{j}^{*} v\left(t_{j}\right)=0 \text { for all } j
$$

Let $v_{1}, \ldots, v_{n}$ be a basis of $V$, then (9) leads with $v=\Sigma a_{\nu} v_{\nu}$ to the system of equations

$$
\sum_{\nu} a_{\nu} x_{j}^{*} v_{\nu}\left(t_{j}\right)=0, \quad j=1, \ldots, m+1,
$$

which has a nontrivial solution $\alpha_{1}, \ldots, \alpha_{n}$, since the rank of the matrix of (10) is at most $n-1$ because of $n \geq m+1$ and (8). Therefore a $v \neq 0$ in $V$ with (9) exists.

If $\|f-\lambda \nu\|=1+\delta$ then $\left\|f\left(t_{j}\right)-\lambda v\left(t_{j}\right)\right\|=1+\delta$ for at least one $j$. From this it follows $\left\|\lambda v\left(t_{j}\right)\right\| \geq K \psi(\delta)$ by hypothesis and so $\|\lambda v\| \geq K \psi(\delta)$.

From Theorem 3 one can obtain a bound for the modulus of continuity of the metric projection similar to that of Corollary 1 . It may be noted that the bound of Corollary 1 is not sharp in general. For a Hilbert-space of dimension $\geq 2$ it yields $\left\|P_{V}(f)-P_{V}(g)\right\|=O\left(\|f-g\|^{1 / 2}\right)$ which is less sharp than the well-known estimate $\left\|P_{V}(f)-P_{V}(g)\right\| \leq\|f-g\|$.

\section{REFERENCES}

1. J. A. Clarkson, Uniformly convex spaces, Trans. Amer. Math. Soc. 40 (1936), $396-414$.

2. $\mathrm{Ky} \mathrm{F}$ an and $\mathrm{I}$. Glicksberg, Some geometric properties of the spheres in a normed linear space, Duke Math. J. 25 (1958), 553-568. MR 20 \#5421.

3. G. Freud, Eine Ungleichung für Tschebyscheffsche Approximations polynome, Act a Sci. Math. (Szeged) 19 (1958), 162-164. MR 21 \#251.

4. S. J. Poreda, On the continuity of best polynomial approximations, Proc. Amer. Math. Soc. 36 (1972), 471-476.

5. A. Schönhage, Approximations theorie, de Gruyter, Berlin, 1971. MR 43 \#3693.

6. I. Singer, Best approximation in normed linear spaces by elements of linear subspaces, Editura Academiei Republicii Socialiste România, Bucharest, 1967; English transl., Die Grundlehren der math. Wissenschaften, Band 171, Springer-Verlag, Berlin and New York, 1970. MR 38 \#3677; 42 \#4937.

INSTITUT FÜR ASTROPHYSIK, MAX-PLANCK-INSTITUT FÜR PHYSIK UND ASTROPHYSIK, 8 MÜNCHEN 40, WEST GERMANY 